\title{
Physiotherapy in the intensive care unit
}

\author{
Begin with the end in mind. (Sean Covey)
}

While physiotherapy has been recommended by scientific societies as integral to the management of critically ill patients, great variation has been reported in the role of the physiotherapist in the intensive care unit (ICU), the service provided and the techniques used. ${ }^{[1]}$ Clearly, this may impact on patient outcome. In a bid to address these variations, ICU physiotherapists have taken the initiative to drive a research agenda and to standardise clinical pathways to facilitate optimal patient outcome.

The long-term sequelae associated with surviving an episode of critical illness are becoming increasingly apparent as the population of ICU survivors grows. The body of literature reporting on the 6- to 12-month outcome of survivors has increased from three publications in 1970 to $\sim 300$ published since $2000{ }^{[2]}$ The majority of studies highlight the poor outcome of survivors. This includes reports of a 59\% 1-year mortality, physical and cognitive impairments and poor health-related quality of life (HRQoL) in survivors of critical illness. Decreased muscle strength, joint stiffness, and decreased exercise capacity are lingering symptoms that persist up to 1 year after ICU discharge, and highlight the reality that decisions made and management options used in ICU affect the morbidity and HRQoL of ICU survivors.

It is recognised that immobility in ICU and time spent on a ventilator are detrimental to long-term patient outcome. Much work has been done to ensure that early mobilisation of ICU patients is feasible and safe, and that patients are liberated from mechanical ventilation in a timely manner. Practices regarding optimal nutrition, sedation, weaning and early mobility have been reported. ${ }^{[1]}$ While a recent review postulates that strategies aimed at improving respiratory muscle function will improve the long-term outcome of survivors, the association between respiratory muscle dysfunction and functional outcomes is unclear. ${ }^{[3]}$ Similarly, despite a focus on facilitating the early mobility of critically ill patients, the effect of these early activities on the outcome of survivors is unclear. A recent trial reported no difference in hospital length of stay or muscle strength at 6 months when comparing in-unit standardised rehabilitation with usual care. ${ }^{[4]} \mathrm{A}$ better understanding of the underlying pathophysiology of post-intensive-care syndrome (PICS), and respiratory muscle dysfunction, is needed for clinicians to develop effective strategies to attenuate the consequences of this syndrome. As rehabilitation experts within the multidisciplinary team caring for ICU patients, physiotherapists are uniquely positioned to drive this research agenda.

Standardising clinical pathways has been advocated to address the observed variations in clinical practice. ${ }^{[5]}$ It has been postulated that these variations could be related to the training of therapists. While all universities in South Africa (SA) provide compulsory training to undergraduate physiotherapy students in the management of critically ill patients, the Health Professions Council of SA does not regulate the content of physiotherapy curricula. The results of a task team that has been working on the establishment of minimum standards for training since 2012 are awaited. As no standards exist for the training of therapists working in critical care environments - nationally or internationally work is being done to inform the development of a competency framework for physiotherapists working in ICU. Ultimately, the framework will be endorsed and used throughout SA ICUs. ${ }^{[5]}$ Through a consensus-building process, SA physiotherapists working in ICU were invited to identify core concepts to include in such a framework. Therapists agreed on six concepts that should be included when formulating minimum standards for physiotherapy clinical practice in SA ICUs. Physiotherapists working in ICU should have the necessary knowledge and skills to understand normal integrated physiology and anatomy and to use clinical reasoning to execute holistic assessments and effective physiotherapy techniques. As we move towards the implementation of minimum standards of physiotherapy practice in SA, the two surveys included in this edition of $S A J C C^{[6,7]}$ provide valuable data on current physiotherapy practice in SA ICUs. The information is valuable as an international benchmark and to determine the training needs of physiotherapists practising in SA units.

Lottering and Van Aswegen ${ }^{[6]}$ aimed to describe the current practice of physiotherapists in SA ICUs. A strength of their study is that they attempted to survey both state and private units. Unfortunately, the response rate was only $33.9 \%$, the majority of respondents were from the private sector (60\%), and practitioners providing service in one province, Gauteng, were over-represented (48\%). Despite the shortcomings, their article provides a clear picture of current physiotherapy practice within SA units. Although the physiotherapy service is aligned with current available evidence, it is clear from the data that work is needed to ensure that therapists incorporate the use of objective outcome measures into daily practice. Using the Medical Research Council scale to identify patients presenting with PICS before ICU discharge could facilitate targeted rehabilitation of these patients. In addition, the collection and reporting of objective measures may inform the development of process measurements as quality indicators for physiotherapy services in ICU. ${ }^{[8]}$

In the other survey, Morar and Van Aswegen ${ }^{[7]}$ aimed to establish the extent of involvement of SA physiotherapists in the weaning of patients from mechanical ventilation (MV) and describe current physiotherapy practice related to weaning and extubation. The response rate for this survey was $44 \%$ and the majority of respondents were from the public sector (66\%). It is evident from the data that the SA therapists surveyed have limited involvement in the adjustment of MV settings, decision-making regarding initiation of patient weaning, make limited contributions towards the development of weaning protocols for their units and do not participate in the extubation of patients. These results could be explained by the large number of therapists with $<5$ years' experience $(62 \%)$ participating in this survey. It also confirms the need for standardisation of curricula. It is encouraging that the majority of respondents contribute towards weaning of patients from MV through prescription of exercise therapy, early out-of-bed mobilisation and deep breathing exercises. However, the survey highlights the need for further training of physiotherapists on the criteria for patient weaning and additional targeted methods that could be employed to facilitate respiratory muscle training. Both the articles highlight the importance of developing and implementing minimum standards for physiotherapists working in SA ICUs.

Outcomes are the end result of the care that a patient receives. ${ }^{[8]}$ In addition to mortality, morbidity and health-related quality of life have been identified as outcomes that should be used to evaluate the quality of care provided to critically ill patients. As we move beyond mortality as the primary outcome, physiotherapists are positioned as integral members of the multidisciplinary team that will ensure optimal outcome for ICU survivors.

\section{Prof. S Hanekom (PhD)}

Head of Physiotherapy Division, Faculty of Medicine and Health

Sciences, Stellenbosch University, Cape Town, South Africa

sdh@sun.ac.za 
1. Stiller K. Physiotherapy in intensive care: An updated systematic review. Chest 2013;144(3):825847. DOI:10.1378/chest.12-2930

2. Turnbull A, Rabiee A, Davis W, et al. Outcome measurement research from 1970 to 2013: A scoping review of 425 publications. Crit Care Med 2016;44(7):1267-1277. DOI:10.1097/ CCM.0000000000001651

3. Shellekens WJ, Van Hees HW, Doorduin J, et al. Strategies to optimize respiratory muscle function in ICU patients. Crit Care 2016;20(1):103. DOI:10.1186/s13054-016-1280-y

4. Morris PE, Berry MJ, Files DC, et al. Standardised rehabilitation and hospital length of stay among patients with acute respiratory failure: A randomized controlled trial. JAMA 2016;315(24):26942702. DOI:10.1001/jama.2016.7201

5. Hanekom S, Van Aswegen H, Plani N, Patman S. Developing minimum clinical standards for physiotherapy in South African intensive care units: The nominal group technique in action. J Eval Clin Pract 2015;2(1):118-127. DOI:10.1111/jep. 12257
6. Lottering M, Van Aswegen H. Physiotherapy practice in South African intensive care units. S Afr J Crit Care 2016;32(1):11-16. DOI: 10.7196/SAJCC.2016.v32i1.248

7. Morar D, Van Aswegen H. Physiotherapy contributions to weaning and extubation of patients from mechanical ventilation. S Afr J Crit Care 2016;32(1):6-10. DOI:10.7196/SAJCC.2016. v32i1.254

8. Murphy DJ, Ogbonna CO, Coopersmith C. ICU director data: Using data to assess value, inform local change, and relate to the external world. Chest 2015;147(4):1168-1178. DOI:10.1378 chest.14-1567

S Afr J Crit Care 2016;32(1):3-4. DOI:10.7196/SAJCC.2016.v32i1.293 